\title{
Visualization of natural processes applying rough-fuzzy temporal granularity to laser speckle images
}

\section{Visualización de procesos naturales aplicando granularidad temporal difusa rugosa a imágenes de speckle}

\author{
A. L. Dai Pra1 ${ }^{*}$, H. Rabal ${ }^{2}$ \\ 1 Research Group on Artificial Intelligence applied to Engineering.Faculty of Engineering, National \\ University of Mar del Plata, Juan B. Justo 4302, Mar del Plata 7600, Argentina \\ 2 Optical Research Centre, CIOp (CONICET La Plata - CIC) and UIDET Optimo, Basic Sciences Department, \\ Faculty of Engineering, National University of La Plata, P.O. Box 3, Gonnet, La Plata 1897, Argentina. \\ ${ }^{(*)}$ E-mail: daipra@fi.mdp.edu.ar
}

Received: 05/09/2017 Accepted: 21/05/2018

DOI: $10.7149 /$ OPA.51.2.50017

\begin{abstract}
:
We present a methodology to visualize a natural process by applying computational granularity to dynamic laser speckle pattern images. This methodology not only allows to identify activity levels with few images but also to visualise how the activity evolves over time even when alternative techniques do not detect it. Two examples are shown, one relative to detection of bruise in apples and other relative to paint drying time, which are of commercial/industrial interest.
\end{abstract}

Keywords: Temporal computational granularity; Rough-fuzzy sets; dynamic laser speckle; Bruise in apples; Paint drying time

\section{RESUMEN:}

Presentamos una metodología para visualizar procesos naturales aplicando granularidad temporal a imágenes de patrones de moteado laser dinámico. Esta metodología permite identificar niveles de actividad empleando pocas imágenes y además, visualizar como la actividad evoluciona en el tiempo en casos donde otras técnicas alternativas no lo detectan. Se muestran dos ejemplos, uno relativo a la detección de daño en manzanas y otro relativo al tiempo de secado de pintura, ambos de interés comercial e industrial.

Palabras clave: Granularidad computacional; Granularidad temporal; Conjuntos difusos-rugosos; Daño en manzanas; Tiempo de secado en pinturas

\section{REFERENCES AND LINKS / REFERENCIAS Y ENLACES}

[1] H. J. Rabal, R. A. Braga, Dynamic Laser Speckle and Applications. CRC Press, Boca Raton Florida, (2008).

[2] J. D. Briers, "Laser speckle contrast imaging for measuring blood flow," Opt Appl 37, 139-152 (2007).

[3] A. L. Dai Pra, L. Passoni, I. H.Rabal, "Evaluation of laser dynamic speckle signals applying granular computing," Signal Process 89, 266-274 (2009).

[4] C. Weber et al., "Determination of maize hardness by biospeckle and fuzzy granularity," Food Sci Nutr 2, 557-564 (2014).

[5] J. Amalvy, C. et al., "Application of dynamic speckle interferometry to the drying of coatings," Prog Org Coat 42, 89-99 (2001).

[6] M. Neisenbaun et al., "Hidrocarbon biodegradation and dynamic speckle laser for detecting chemotactic responses at low bacterial concentration," J Environ Sci 25, 613-625 (2013).

[7] A. L. Dai Pra et al., "Temporal and Spatial Fuzzy granularity of laser and ultrasound frames," VI Latin American Conference on Biomedical Engineering, October 2014. Paraná. Entre Ríos. Argentina, (2014). 
[8] E. Todorovich et al., "Real-time speckle image processing," J Real-Time Image Proc. 11, 535-545 (2016).

[9] J. M. López-Alonso et al., “Dynamic speckle analysis using multivariate techniques," J Opt 17, 035609035616 (2015).

[10] W.Pedrycz, “Granular Computing: An Emerging Paradigm,” Phisica- Verlag, Germany, (2001).

[11] Y.Yao, "Partition Model of Granular Computing. Transactions on Rough Sets I," Lect Notes Comput Sc 3100, 232-253 (2004).

[12] L. A. Zadeh, "Toward a theory of fuzzy information granulation and its centrality in human reasoning and fuzzy logic," Fuzzy Sets Syst 90 111-127 (1970).

[13] A. Bargiela, W. Pedrycz, "Granulation of temporal data: a global view on time series," in: IEEE Proceedings of the 22nd International Conference of the North American Fuzzy Information Processing Society. 191-196 (2003).

[14] W. Pedrycz, A. Gacek, “Temporal granulation and its application to signal analysis," Inf. Sci. 143, 47-71 (2002).

[15] Pal, S. K., "Granular Mining and Rough-Fuzzy Pattern Recognition: A Way to Natural Computation," IEEE Intelligent Informatics Bulletin December 2012, 3-13 (2012).

[16] P. Maji, S. K. Pal. Rough-fuzzy pattern recognition. Applications in bioinformatics and medical imaging. John Wiley \& Sons, NY. (2011).

[17] S. Greco, B. Matarazzo, R. Słowinski, "A summary and update of "Granular Computing and Data Mining for Ordered. Data: the Dominance-based Rough Set Approach”. In: R.A. Meyers (ed.), Encyclopedia of Complexity and Systems Science. Springer, New York, 4283-4305. (2009).

[18] L. A. Zadeh, "Fuzzy sets," Inform Control 8, 338-353 (1965).

[19] D. Dubois, H. Prade. Fuzzy Sets Systems. Academic Press, New York. (1980).

[20] Z. Pawlak, “Rough sets,” Int J Comput Inf Sci 11, 341-356 (1982).

[21] Z. Pawlak, A.Skowron, "Rudiments of rough sets," Inform Sciences 177, 3-27 (2007).

[22] R. Jensen, Q.Shen, "Fuzzy-rough sets for descriptive dimensionality reduction". Fuzzy Systems 1, 29 34 (2002).

[23] E. Hecht, A. Zając. Optics. Reading, Massachusetts, Addison-Wesley Pub. Co. (1974).

[24] A. L. Dai Pra et al., "Signal Feature Extraction Using Granular Computing. Comparative Analysis with Frequency and Time Descriptors Applied to Dynamic Laser Speckle Patterns," Int J Comput Int Sys 8, 28-40 (2015).

[25] W. Pedrycz, "From numeric models to granular system modelling," Fuzzy Inf. Eng 7, 1-13 (2015).

[26] A. K. Dunn, "Laser Speckle Contrast Imaging of Cerebral Blood Flow," Ann Biomed Eng 40, 367-377 (2012).

[27] R. Nassif et al., "Detection of Golden apples climacteric peak by laser biospeckle measurements," Appl Optics 53, 8276-8282 (2014).

[28] L. I. Passoni et al., "Dynamic speckle processing using wavelets based entropy,” Opt Commun 246, 219 228 (2005).

[29] A. L. Dai Pra, L. I. Passoni, H. Rabal. "Fuzzy Granular Computing and Dynamic Speckle Interferometry for the Identification of Different Thickness of Wet Coatings," INFOCOMP 8 (4), 45-51 (2009).

[30] U. L. Opara, P. B. Pathare. "Bruise damage measurement and analysis of fresh horticultural produce-A review," Postharvest Biol Technol 91, 9-24 (2014).

[31] G. ElMasry, N. Wang, C. Vigneault. "Detecting chilling injury in Red Delicious apples using hyperspectral imaging and neural networks," Postharvest Biol Technol 52, 1-8 (2009).

[32] R. Lu, "Detection of Bruises on Apples Using Near-Infrared Hyperspectral Imaging," Trans.ASAE 46 523-530 (2003).

[33] L. Yuzhen, L. Richard, L. Renfu, "Detection of fresh bruises in apples by structured-illumination reflectance imaging," Proc. SPIE 9864, Sensing for Agriculture and Food Quality and Safety VIII, 986406, May 17, (2016).

[34] M. Pajuelo et al., "Bio-speckle assessment of bruising in fruits," Opt Laser Eng 40, 13-24 (2003).

[35] T. N. Dang, L. Wilkinson, A. Anand, “Stacking Graphic Elements to Avoid Over-Plotting," IEEE T Vis Comput. Gr 16, 1044-1052 (2010) 


\section{Introduction}

When a beam of coherent light (laser) illuminates an object with a surface that is rough in comparison with the wave length, the light is scattered in all directions and an interference pattern of granular or noisy aspect, called 'speckle pattern', can be observed. It consists mainly in a generalized Doppler effect. Light scattered in the medium suffers changes in its instantaneous frequency due to the movement of the scattering centres or to any other time variations in optical path. Frequency shift wavelets beat their contributions on the detector producing low frequency intensity variations. Thus, when the objects have some type of surface activity, such as in biological specimens or certain physical/chemical phenomena, the particles on the surface move and the speckle pattern changes along time [1].

Dynamic speckle has permitted to obtain important information relative to the dynamics of the objects under study in a non-invasive way. It has been used to monitor blood flow and other tissue movements in the body [2]; to screen viability and endosperm proportions or different characteristics in seeds [3, 4]; to control paint drying time [5]; to observe bacteria movements [6], to improve ultrasound medical images [7] and others.

Stacks of successive images are obtained with CCD cameras with a suitable resolution to register the phenomenon; then, the intensity change in every pixel along time permits the analysis of activity degrees in each point of the observed objects. The intensity changes can be considered as time series and different techniques of signal processing can be used to obtain values that describe its behaviour. With the set of values (descriptors) obtained for every pixel, an image is made up where it is possible to visualise different activity characteristics.

Some techniques do not allow the detection of certain activities, and many of these require a high number of images to provide good results. In some cases, the time of evolution of the analysed activity is not known a priori and changes occurring inside the time required for the register are lost. Also, records taken beyond the end of the studied events reduce the efficacy of the analysis because of the recording of assumed activity that has already finished, changed or reduced, and non-stationary phenomena are pooled together and cannot be detected.

A new technique that has been applied with good results is fuzzy temporal granularity. It provided satisfactory results to visualize regions with different activity [3]. The method was simplified by incorporating rough sets concepts, with the aim of being implemented in digital systems [8]. The new version has lower computational complexity and produces the named Rough-Fuzzy Granular Descriptor (RFGD). It can identify activity with few images and therefore perform almost real-time analysis of unquestionable importance in the inspection of biological, physical and/or chemical processes.

In this work, the evolution of natural processes is analysed through RFGD applied to dynamic speckle patterns. The purpose of this work is to show how using speckle laser and RFGD, the evolution of natural processes can be detected and monitored. In section 2, the methodology for acquiring speckle patterns and use of the RFGD are presented. In section 3, two experiments are shown, one biological, such as the early detection of a non-visible bruise in an apple, and other physicochemical, such as the evaluation of paint drying time, through the observation of a surface topography hidden under wet paint. Section 4 discusses the results.

\section{Methodology}

\section{2.a. Speckle pattern}

When laser light illuminates a diffuse surface, the high coherence of the light produces a random interference effect or speckle. In many imaging systems, the speckle is a source of noise that is desirable to eliminate. It has been found that about a $70 \%$ of the speckle energy is composed by noise [9]. However, the dynamics of the speckle pattern contains information about the motion of the scattering particles and other phenomena that introduce changes in optical path in the sample.

To register the speckle pattern, an adequate setup is designed to produce well-resolved speckle grains occupying several pixels in a CCD camera (Figure 1a). For the experiments used in this work, a low power expanded He-Ne laser (10 $\mathrm{mW}$ power, $\lambda=633 \mathrm{~nm}$ wavelength) was used to illuminate a wide region of the sample. The images were formed by a lens with high values of diaphragm opening $(f=50 \mathrm{~mm}, \mathrm{f} / \#=16)$. 


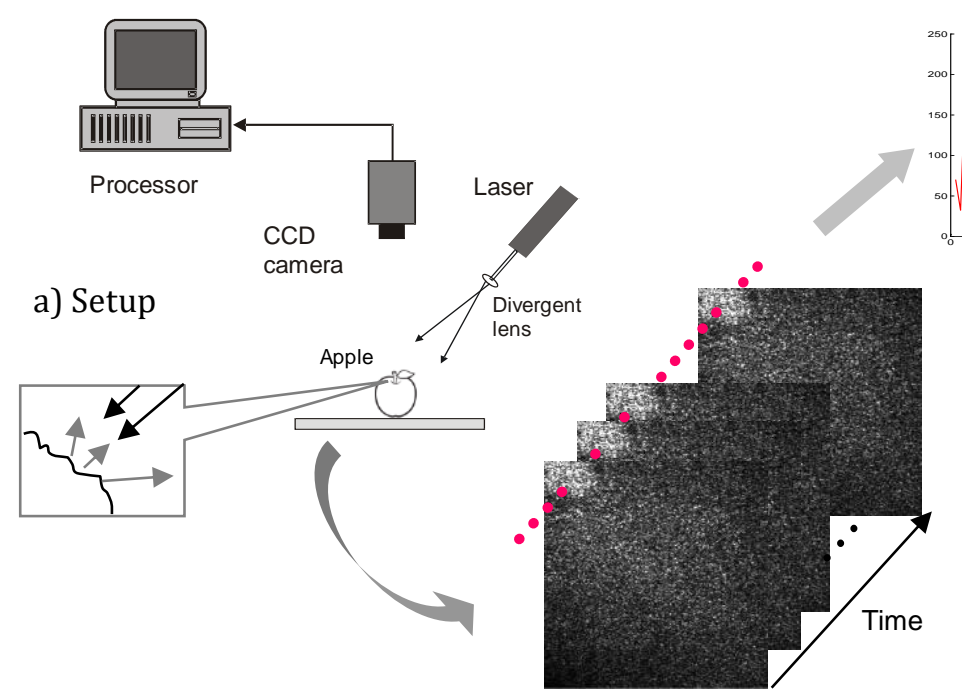

c) A pixel time series

b) Speckle images

Fig.1. Activity detection process

Figure 1 shows a complete activity detection process employing techniques of signal processing: a) is the optical set-up; b) shows a series of speckle pattern images over time (Nowadays the acquisition time is 200 images per second); c) time series are obtained at every pixel through the images, the time series are processed with some technique of signal processing and a value which characterizes the series is obtained (activity descriptor). d) With the obtained descriptor values, an image is reconstructed, where the intensity of each pixel corresponds to the activity value descriptor.

\section{2.b. Rough-fuzzy granularity}

Granular computation is a process of abstraction that permits to simplify the complexity of the information. As any abstraction, it intends, in terms of human perception, to facilitate the comprehension of some physically complex phenomenon.

The granular computation is associated with the group of entities (e.g. numbers, pixels of images, values of time series) in blocks, with the purpose of simplifying the prediction, interpretation and classification of complex problems [10-12].

The Information Granulation in time, leads to the notion of defining time intervals, in which a temporal signal, traditionally represented by a data set, can be transformed and reduced to a set of granules of information to facilitate its analysis, interpretation or classification $[13,14]$. The objective in this work is to evaluate in an image sequence, time intervals in which a pixel keeps a value somewhat similar, defining each of these intervals as a temporal information granule.

Fuzzy sets and rough sets lead to a more general theory of granulation with many potential applications. Rough sets deal with the indiscernibility of objects in relation to their attributes and fuzzy sets deal with the vagueness of expressions with which different object attributes are catalogued [15-17].

Both sets are considered in the RFGD approach.

A fuzzy set is a set whose elements can belong partially to one set and simultaneously to another; this allows vague concepts to be defined $[18,19]$.

A rough set is an approximation of a vague concept by a pair of precise concepts. Rough sets are based on the fact that an object cannot always be defined in precise form (crisp) inside a category on the basis of the value of its attributes.

Formally a rough set is expressed as: 
Given an information system $S=(U, A)$, with $U$ the universal set defining all the objects to consider, with subsets $X \subseteq U$; $A$ the set defining the possible attributes of the $x$ objects, with subsets $B \subseteq A$; and an equivalence class $[x]_{B}$ which defines a relation in which elements in $X$ are indiscernible from each other by attributes from $B$; a rough set is defined by the sets $\left\{x \in U:[x]_{B} \subseteq X\right\}$ and $\left\{x \in U:[x]_{B} \cap X \neq \varnothing\right\}$ which are denominated as $B$-lower approximation and B-upper approximation of $X$ in $B$ and denoted as $\underline{B} X$ and $\bar{B} X$ spectively. The objects in $\underline{B} X$ certainly possess the $B$ attribute and the objects in $\bar{B}$ X possibly or partially possess the $B$ attribute $[20,21]$.

If $B$ is a fuzzy attribute, the sets are rough-fuzzy sets [22].

\section{2.c. RFGD calculation}

When a speckle pattern is captured, the resulting images contain grainy high-contrast structures. The measured intensity in each pixel is a result of the interference of Huygens secondary wavelets [23]. All have travelled different path lengths from the optically rough surface to the camera, so that their relative phase's differences are random. For the detection of speckle motion, each pixel intensity change is evaluated as time series. These time series are noisy and dependent on the observed sample and the optical employed setup.

The RFGD evaluates activity of different pixels independently of the knowledge of the studied phenomenon. Some of the descriptors normally used, described in the literature [1], are adequate for particular cases, but RFGD so far has provided good results in all cases that it was applied [24]. In general, there is not a standard or pattern of comparison for the results; they depend on what expects to be observed or it is evaluated by an expert in the problem, considering the sample and the proposed aim. So basically the intention is to discover information not perceptible to the human eye, generally, without a gold standard.

In this application it was considered to distinguish initially three possible states of pixel intensity: dark, medium and light, these states are subjective and overlapped, and they can be easily defined with roughfuzzy sets. Information granules are formed in function of these states and the quantity of granules in time can be tested as a measure of activity.

Formally, a time series $T S_{(x, y)}$ is obtained for each pixel $(x, y)$ in a sequence of intensity images. It represents the intensity variation of a pixel in time (Figure 1c). The aim of RFGD is to turn the $T S_{(x, y)}$ series into a set of granules of information characterised by shades of grey. In this way, small variations of intensity are disregarded and the granules quantification is adopted as an activity descriptor.

Other characteristics or attributes of the granules as frequencies, size, distribution, might be analysed to study other particular characteristics of the series, not considered in this particular case.

Considering the universal set of intensity values $U=[0,255]$ and the attribute $A$ corresponding to shades of grey, three fuzzy concepts $F_{k} \subseteq A$, with $F_{k}=\{$ dark, medium and light $\}$, are selected for the $V$ set of intensity values of an image, with $\{v \in V, V \subseteq U\}$. The grey levels in $V$ are dependent on the image characteristics, such as range, brightness and contrast.

In order to determine an adequate coverage of the information granules [25] and ensuring that they should not be affected by the image characteristics that could overshadow the discernment between the different granules, an intensity histogram $H$ of 255 bins of a single speckle image is evaluated. It is considered that the intensity distribution is very similar for all images of the sequence. The range of intensity values is then divided into five regions $R_{h}$ with similar cardinality (card) (fig. 2a)), the extreme and central ones correspond to totally certain values for the $F_{k}$ fuzzy sets (lower approximation); the second and fourth ones correspond to boundary regions that are not totally certain for the $F_{k}$ fuzzy sets and are common to more than one set (fig. $2 b$ )).

Considering the granule coverage (cov) in a image as in Eq. 1, where ir and ic are the number of rows and columns respectively, the dark and light granules have similar cover and the medium granules have slightly higher coverage for including two boundary regions; it is expected that the pixels belonging to this last granule class correspond to blurry zones associated with fluctuations of intensity faster than the time of the capture of the camera [26]. After different considerations this coverage distribution of fuzzy concepts provided the most satisfactory results. The proposed distribution was achieved analysing the changes in different series, observing behaviours and then automating it.

$$
\operatorname{cov}\left(F_{k}\right)=\frac{1}{i r^{*} i c} \operatorname{card}\left(T S_{(x, y)}(j) / T S_{(x, y)}(j) \in F_{k}\right), \quad j=1, n
$$


To granulate a $T S_{(x, y)}$ of length $n$, successions of equal equivalence classes $[v]_{F k}$ are considered (upper approximation). A granule ends when a pixel in the succession changes its equivalence class. The granules definition is held simultaneously for the three sets, since the granules can be partially or totally overlapped.

Being $I$ an image of ir $\mathrm{x}$ ic and $n$ the images quantity, for each $T S_{(x, y)}$ the end of the each one of the $k$ granules in each $j$ instant (or image) is verified and stored in a Gr matrix of ir x ic $x k$, where 1 indicates the end of a granule and 0 indicates continuity or change of granule class (start of a new granule) (eq. 2).

$$
G r_{j}(x, y, k)=\left\{\begin{array}{c}
1 \text { if }\left[T S_{(x, y)}(j-1)\right]_{B_{k}}=\text { true and }\left[T S_{(x, y)}(j)\right]_{B_{k}}=\text { false } \\
0 \text { in othercase }
\end{array}\right.
$$

The RFGD matrix of ir x ic accumulates the $G r$ values of each image. That is, how many granules end in each instant $j$ for each pixel $(x, y)$. A relative value can be computed for $n$ images (eq. 3).

$$
R F G D_{n}(x, y)=\left(\sum_{j=2}^{n} \sum_{k=1}^{3} G r_{j}(x, y, k)\right) / n
$$

Figure 2 shows the methodology as applied to arbitrary $T S_{(x, y)}$.

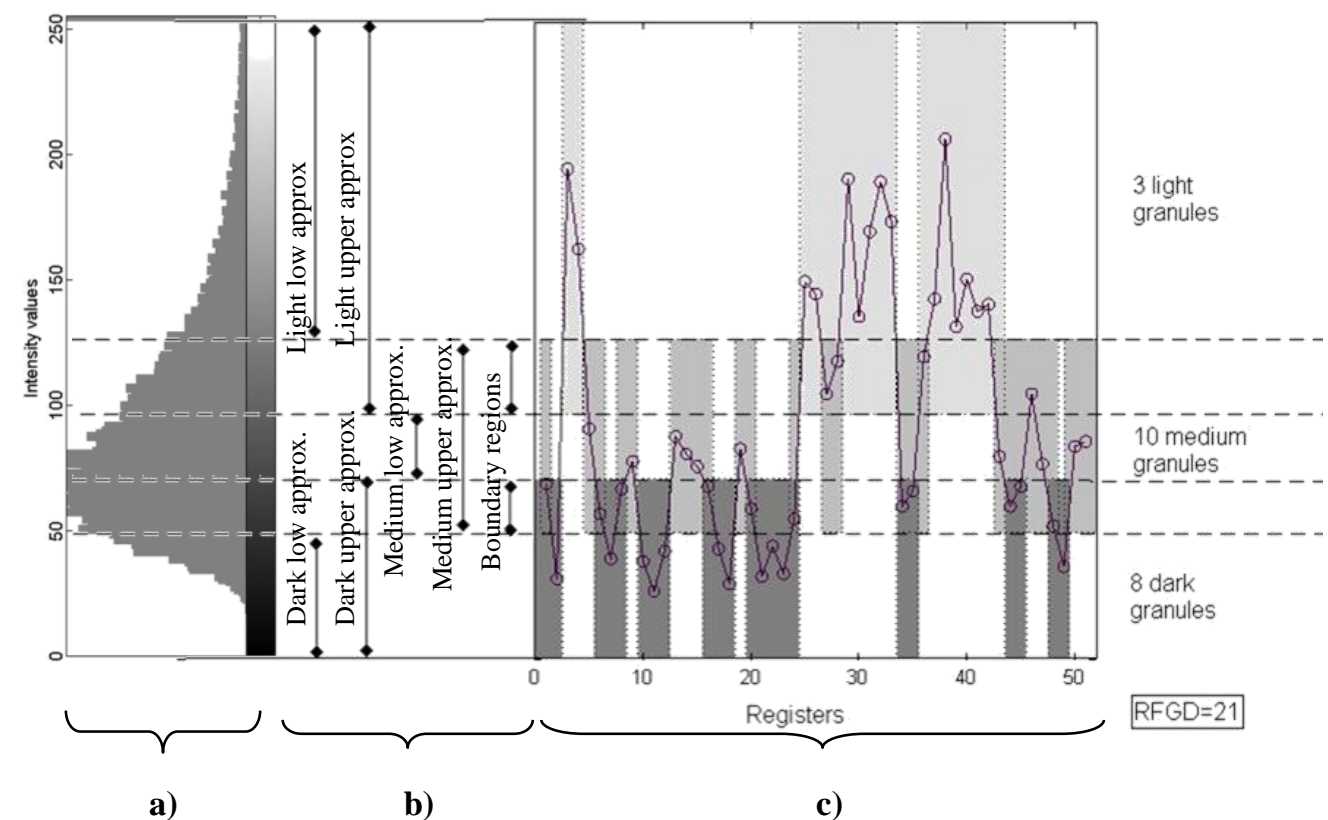

Fig. 2. RFGD process. a) Histogram of one image in grey scale, b) rough approximation c) granulated time series for one arbitrary pixel.

The activity can be calculated in a fixed period of time [3], as is shown in fig. 2, or in real time, evaluating each new image and relating this result to the granules state in previous time [7].

The RFGD matrix obtained permits to identify changes in each $T S_{(x, y)}$, reducing sensitivity to interferences or small variations of contrast and the visualization of a normalized RFGD to intensity pixel values allows to distinguish the points with different activity levels without suffering reduction in spatial resolution, unlike other methods [1], which turns it into a robust algorithm.

\section{Experiment}

In this case two sets of speckle images are analysed, which have already been studied with different methods [1]. It permits to compare the proposed method results with other activity descriptors using the same optical setup and samples.

The images correspond to bruise in apples $[27,28]$ and drying state of paint on a hidden surface $[3,5,29]$. 
These two samples are characterized for having different answers with different methodologies, whereas the methodology proposed here offers the expected results in both cases and requires a much smaller number of frames. So, both register and processing times are reduced.

\section{3.a. Bruise in apple}

Apples are easily struck during the stage of packing and movement and, if the skin is not damaged, the blow is visualized only some days later degrading the quality of the product in the sale stage. Tissue breakage or failure occurs at a microscopic level, causing defects in cells below the skin without damaging it. Breaking the skin or bruising the flesh releases enzymes that in turn cause browning of the fruit $[24,30]$. The bruise becomes perceptible a few days later so it is crucial to detect it early to provide better fruits for the consumer and reduce potential economic losses in the fruit industry.

Different techniques were studied to detect the blow in early stages, such as: hyperspectral imaging [31, 32], structured-illumination reflectance imaging [33] and others, which are not easily reproducible in a sample with the purpose of obtaining comparative results.

For this study, 500 images of speckle patterns of a bruised apple, already used in the analysis of other descriptors, was used. They were obtained immediately after a mechanical damage caused by a controlled impact produced by letting a steel ball fall (diameter: $21.9 \mathrm{~mm}$, weight: $133.6 \mathrm{~g}$.) from $20 \mathrm{~cm}$ height on the apple as in [34]. The illuminated area of the sample in this case is about $5 \mathrm{~cm} \times 5 \mathrm{~cm}$. The bruise images were also registered half an hour later, one hour later and one day later; the intention of this experiment was to see if it was possible to detect some change in the activity before the blow is visible. Every set of images was registered for approximately 6 min.

The speckle pattern of bruise in apples has already been analysed with different methods $[1,24,27]$, with the aim to distinguish damaged regions. As biological samples bear inherent variability we have used the same data obtained for the bruising of the apple that were previously employed for testing other methods to permit the easy comparison with the results shown in the bibliography. Some of these methods allow good differentiation of the damaged regions, but they do not make it possible to detect the blow point along time and to evaluate the activity evolution in the region, which is the intention of this work.

\section{3.b. Paint drying state}

In this experiment the objective is to differentiate the drying time of paint in a sample painted with different thickness and show the activity variation. For this purpose, a 10 cents coin of Argentine peso was covered with a layer of fast drying enamel levelling the surface [29] hoping that the different degrees of activity, due to the thickness of the paint, could allow revealing the topography of the coin, showing its hidden bas relief. On the other way, observation of the topographic details of a coin under paint is an experiment that can be easily reproduced as paint has a predictable behaviour.

\section{Results}

RFGD can obtain relative activity values of the whole images set or estimate it image to image as in a real time process.

The results were analysed by means of 2D and 3D scatter graphs, but as the representation of all pixels at all times was confusing and computationally slow because of data volume $(300 \times 300 \times 500)$, the scatters were reduced in time by computing RFGD every 20 images and, in space, by applying a mean filter in square windows (10x10 pixels), thus avoiding over-plotting [35]. The diminishing of the scatters size provided this way better visualisation of the results.

The 2D scatter graphs allow the activity visualisation in a given time period and the 3D scatter graphs allow the visualisation of the evolution of experiment in time.

The colours of scatters were chosen according to a colour-map representing the activity magnitude (RFGD value). In the 2D case, filled circles were used and, in the 3D case, empty circles were used for transparency.

\section{4.a. Bruise in apple}

Early detection and diagnosis of bruised in apples is rather difficult when the skin is not damaged. Bruises may appear almost immediately or take several days to develop [32]. The RFGD applied to dynamic speckle images can be used in quasi real time because the algorithm is very simple and it can be implemented easily 
in digital systems [7] and may be a tool useful in the early detection of bruise in apples. The acquisition time of 500 images is approximately $2.5 \mathrm{sec}$.

In Figure 3 the 2D scatter graphs show the results of applying the RFGD to the speckle images of samples obtained immediately after the mechanical damage. RFGD was computed every 20 registers as indicated by the number over each graph. The blue region in the corner is a metal inert pointer included to indicate where the bruise was located. The red region is where the bruise and the maximum activity occurred. The red region is present in the first 100 records and then begins to fade, remaining perceivable for a short time.

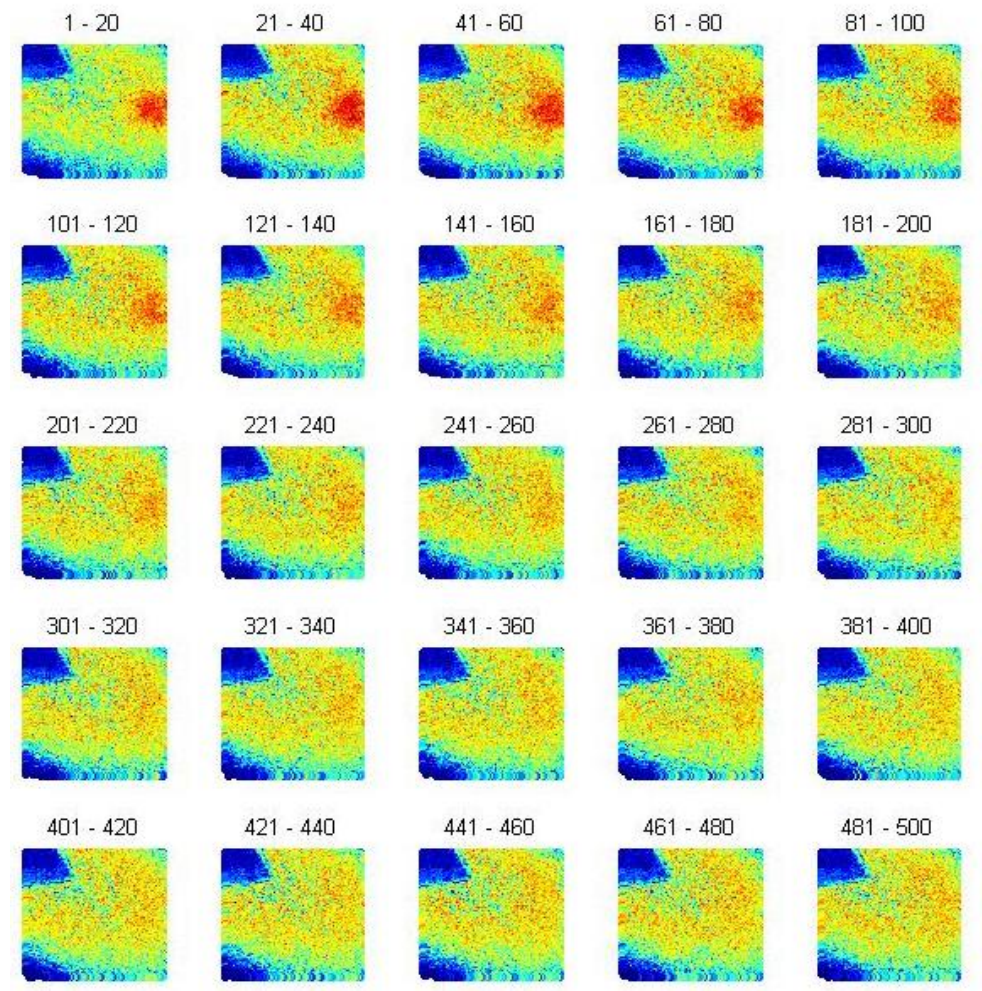

Fig. 3. RFGD results for every 20 speckle patterns

In a 3D scatter graph obtained immediately after mechanical damage (Fig. 4 a), the red region is observed at first and then it reduces later to only a point of inactivity, which coincides with the point of the bruise, is visible. The point remains visible half an hour and one hour later (Fig. $4 \mathrm{~b}$ and $4 \mathrm{c}$ ) and tends to disappear one day after (Fig. $4 \mathrm{~d}$ ) when the firmness of the apple starts to change, but changes of activity were not detected in the rest of the region.

These effects could not be appreciated with other techniques. With RFGD it was possible to detect the evolution of the process and to demonstrate that the impact is detectable only for a brief time. For this reason many of the methods used before could not detect the blow. The use of great quantity of images blurred the initial activity. In addition, the 3D graph allows identifying the impact point because it is aligned in a border. It is not visible in 2D images because of its small size and location. Notice that this way to show the results permits easily the observation of any cut (plane or not) in the space-time volume.

This feature was not noticed with previous techniques, because it was not possible to perform comparative analysis among evaluations obtained in short periods. The time required to record the activity images had to be considered only after using this methodology. The times of each stage can be calculated in terms of register intervals. 


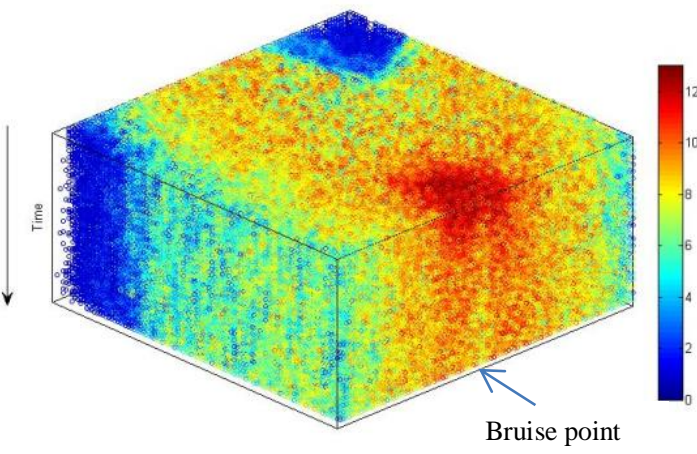

a)

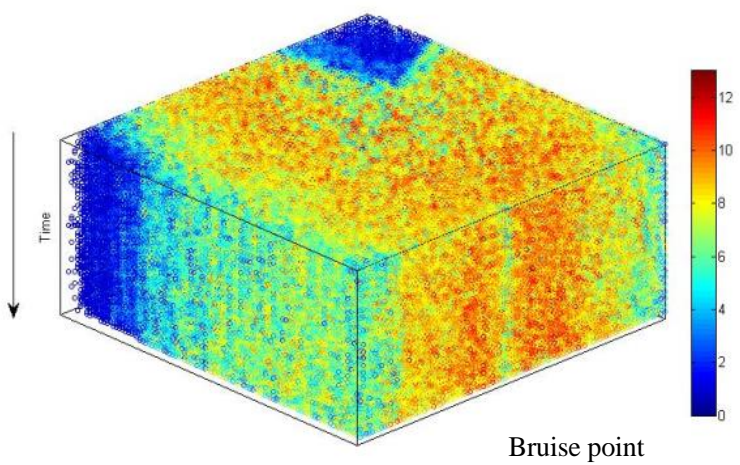

c)

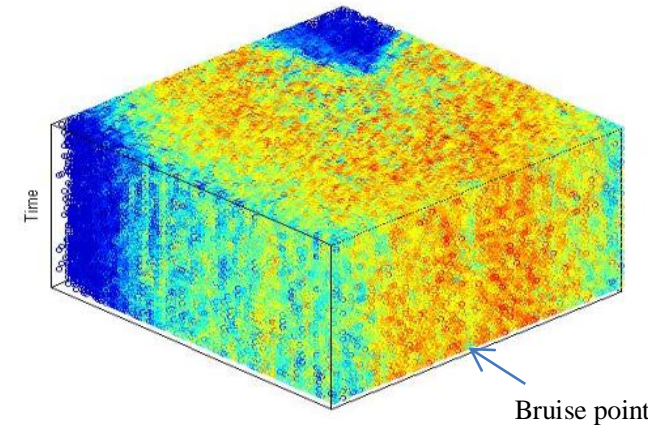

b)

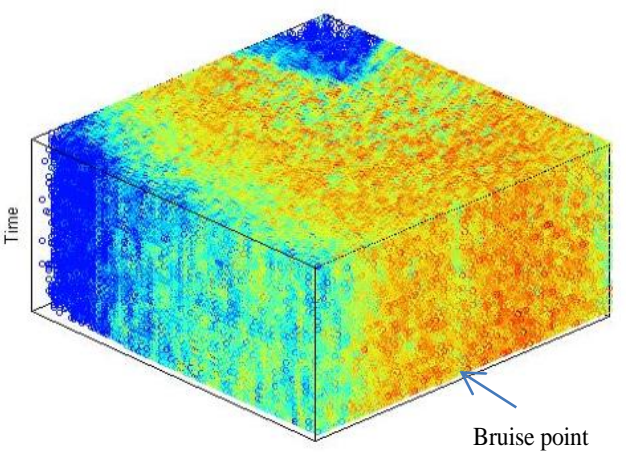

d)

Fig. 4. Results along time: a) immediately after; b) half an hour later; c) one hour later; d) one day later.

Fig. 5 shows results obtained for the immediately after bruise stage, with RFGD and other traditional methodologies [1, 24] as: autocorrelation and standard deviation (statistical), average differences (temporal) and energy of spectral band, Shannon entropy, cutoff frequency, mean frequency and wavelet entropy (frequency). In the processing of the first 20 and all together 500 speckle patterns, some of these cannot to detect the bruise and others do not make a clear difference between the bruised and not bruised regions. In the processing of all together speckle patterns RFGD continues marking clearly the regions and the bruise point. It is necessary to consider, that in addition, the descriptors based on frequency, require much longer processing time.

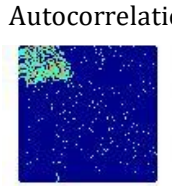

Fuzzy Granularity Spectral Band Energy

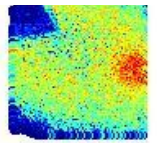

Cutoff Freq.
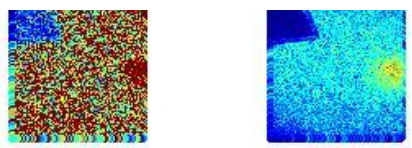

a)

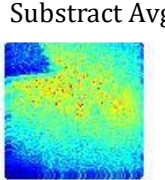

Shannon Entropy

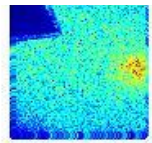

Wavelet Entropy

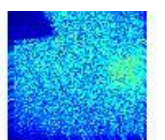

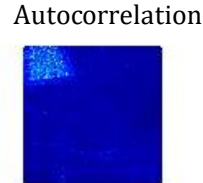

Fuzzy Granularity

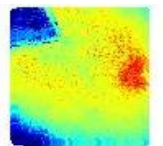

Cutoff Freq.

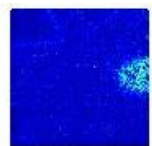

Std. Desv

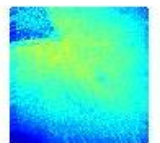

Spectral Band Energy Shannon Entropy

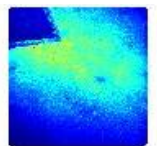

Mean Freq.

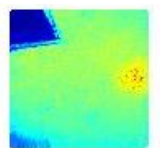

b)
Substract Avg.
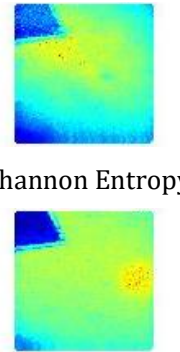

Wavelet Entropy

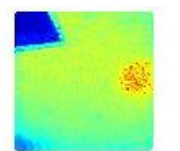

Fig. 5. Results obtained immediately after mechanical damage with RFGD and others traditional methodologies a) for the first 20 speckle patterns, b) for all 500 speckle patterns 


\section{4.b. Paint drying state}

The result of this experiment is shown Fig. 6. The RFGD already provides results with a few registers. In the painted coin experiment, the coin was perfectly distinguishable with only 50 registers. It shows the difference of drying in layers of different thickness.

The resulting image reproduces the topography of the coin not visible in the speckle pattern images. The darkest or blue tones are regions of the highest relief, which correspond to the thinnest layer of paint. This one is the driest zone or of lower activity. The brightest or red regions are topographic depressions that indicate areas with a thicker layer of paint, more humid and therefore more active. In addition, it was also possible to observe that with 200 registers the results were not already improving, the painting had almost dried, activity was diminishing and the speckle patterns were not offering new information, but deteriorating the results obtained with a smaller number of images because of the decreased activity.

In fig $6 \mathrm{~b}$ ) it is possible to observe how the different thickness layers are drying; the numbers indicate the range of registers evaluated. The red colour indicates high activity and the blue low activity. This particularity allows, then, to use the RFGD to evaluate the time of drying of paint depending on the thickness of the layer of paint.

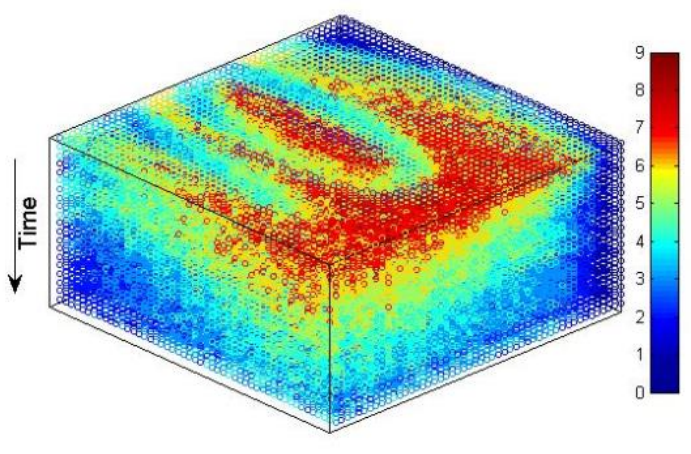

a)

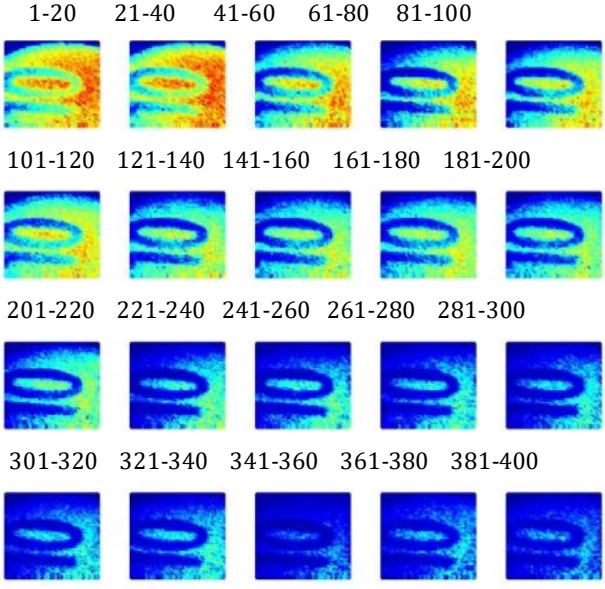

b)

Fig. 6. RFGD Results along time of paint drying

Figure 6a) shows the results in 3D scatter graph, where the evolution of the drying is visualized.

Figure 7 shows the results of paint drying applying different methodologies, as in Fig. 5. In this experiment it is noticeable that some descriptors lose definition when they include periods with low activity in the process (fig. 7 b)). RFGD detects inclusive small blemishes in the contour of the coin.

\section{Conclusions}

Granular computing, based on fuzzy and rough sets, allows a simple, fast and effective processing of time series obtained by the variation of every pixel in a stack of speckle images.

The RFGD algorithm facilitates the processing of dynamic speckle patterns, allowing results in quasi real time thanks to the simplicity of the algorithm, its robustness due to less noise sensitivity and its need of only a few images. The method is faster than those based in frequency [24], in speed it is possible to compare favourably with time domain methods, though these have not given satisfactory results in all the tests as RFGD did.

Used together, dynamic laser speckle and RFGD can be a suitable non-destructive tool for the detection of processes where there are superficial regions with different activity, without suffering reduction in spatial resolution. 
The 3D scatter graph of reduced information permits the observation of the evolution of some different physicochemical or biologic activities over time, permitting better visualisation than that obtained in 2D graphics.

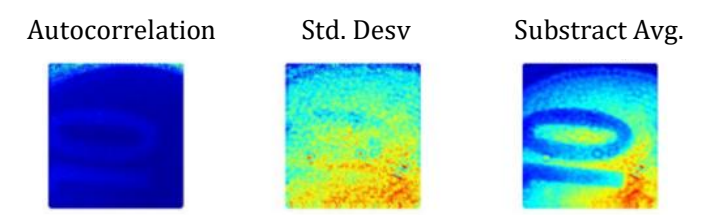

Fuzzy Granularity Spectral Band Energy Shannon Entropy

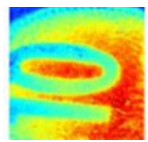

Cutoff Freq

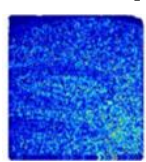

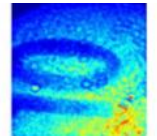

Mean Freq.

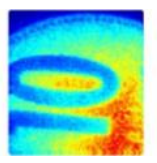

a)

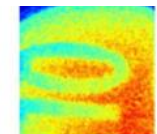

Wavelet Entropy

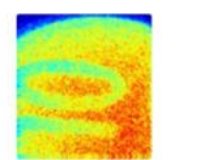

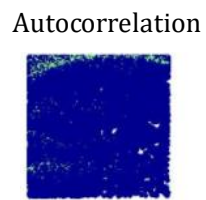

Fuzzy Granularity

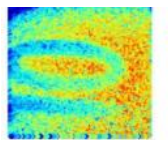

Cutoff Freq.

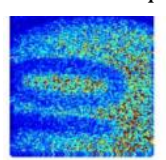

Std. Desv

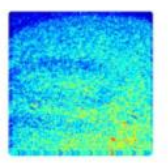

Spectral Band Energy

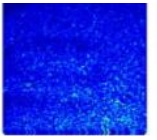

Mean Freq.

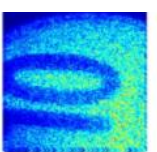

avelet Entropy

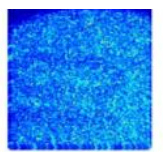

b)

Fig. 7. Results obtained for paint drying with RFGD and others traditional methodologies

a) for the first 20 speckle patterns, b) for 500 speckle patterns

The bruising in the apple is identified in the first stages of the process. A small region at the centre of the bruising, undetected by other techniques, remains visible for a longer time.

New studies could be performed for apples in special conditions (temperature, inoculation and time) and for others biological / physicochemical process.

In the experiments with paint drying time, different existing descriptors did not behave accurately. This is due to the fact that many images are required and the first images of the stack were in a different drying state than the later, so that different activities were pooled in a single result. By using the procedure described here, as it requires a significant smaller number of images, better resolution in time was possible to find smaller differences in the drying state.

RFGD's potential is being studied for other cases of speckle as in ultrasound images used for the detection of malignancy in tumours and other characteristics [7]. In this case, the almost real time feature could be important to reduce the blur in the activity images due to involuntary movements.

\section{Acknowledgements}

This research was supported by a grant CONICET PIP № 112-201101-00520 and by CICBA (Argentina). 